\title{
Experimentally induced cartilage degeneration treated by pulsed electromagnetic field stimulation; an in vitro study on bovine cartilage
}

Francesca Veronesi ${ }^{1 *}$, Milena Fini $^{1,2}$, Gianluca Giavaresi ${ }^{1,2}$, Alessia Ongaro $^{3}$, Monica De Mattei $^{3}$, Agnese Pellati $^{3}$, Stefania Setti ${ }^{4}$ and Matilde Tschon ${ }^{1,2}$

\begin{abstract}
Background: Osteoarthritis $(\mathrm{OA})$ is the final result of progressive alterations to articular cartilage structure, composition and cellularity, followed by an increase in the concentration of pro-inflammatory cytokines in joint synovial fluid. Even though the effect of pulsed electromagnetic field (PEMF) stimulation in counteracting OA progression and inflammation is of increasing interest, because of its anabolic and anti-inflammatory properties, the present study aimed to improve the knowledge on cartilage extracellular matrix (ECM) and chondrocyte changes related to the exposure of PEMF, from a histological and histomorphometric point of view.
\end{abstract}

Methods: An in vitro OA model was realized, culturing bovine cartilage explants with a high dose of interleukin $1 \beta(I \mathrm{~L} 1 \beta, 50 \mathrm{ng} / \mathrm{ml})$ at different experimental times (24 h, and 7 and 21 days). The effects of PEMFs $(75 \mathrm{~Hz}, 1.5 \mathrm{mT})$ were evaluated in cartilage explants treated with IL $1 \beta$ or not (control), in terms of cartilage structure, cellularity and proteoglycans, glycosaminoglycans, collagen II and transforming growth factor $\beta 1$ synthesis by using histology, histomorphometry and immunohistochemistry.

Results: Making a comparison with control cartilage, IL1 $\beta$-treated explants showed a decrease in cartilage matrix, structure and cellularity parameters. PEMFs were able to counteract the progression of OA acting on both cartilage cellularity and ECM in cartilage previously treated with IL1 $\beta$. Normal distribution (Kolmogroc-Smirnov test) and homoscedasticity (Levene test) of data were verified, then, the non-parametric Kruskal Wallis test followed by Mann-Whiteny $U$ test for pairwise comparisons were performed. The $p$-value was adjusted according to the Dunn-Sidak correction.

Conclusions: These results, obtained by culturing and treating cartilage explants from two different joints, confirmed that PEMF stimulation can be used as adjuvant therapy to preserve cartilage from detrimental effects of high inflammatory cytokine levels during OA.

Keywords: Osteoarthritis, Cartilage, Explants, Inflammation, Microenvironment, Pulsed electromagnetic field

\section{Background}

The presence of pro-inflammatory cytokines in the joint microenvironment favors cartilage degeneration and osteoarthritis (OA) progression with alterations of proteoglycans (PGs) and collagen fibers, prevalently collagen type II (Coll II) [1]. Among pro-inflammatory

\footnotetext{
* Correspondence: francesca.veronesi@ior.it

'Department Rizzoli RIT, Rizzoli Orthopedic Institute, Laboratory of Biocompatibility, Innovative Technologies and Advanced Therapies, Bologna 40136, Italy

Full list of author information is available at the end of the article
}

cytokines, interleukin1 $\beta$ (IL1 $\beta$ ) plays a pivotal role in inducing degradation of extracellular matrix (ECM) components and synthesis of other pro-inflammatory cytokines, chemokines and proteolitic enzymes. Since cartilage has a poor intrinsic reparative capability if not treated, OA leads to progressive disability and requirement of joint arthroplasty [2].

Current therapeutic strategies to prevent OA include the use of nonsteroidal anti-inflammatory drugs (NSAIDs) [3], that act at articular and not cellular levels, intraarticular injection of hyaluronic acid (HA) and physical 
exercises [4], that act only in the muscle of the joint. These treatments relieve pain and inflammation and improve functionality, but do not resolve the pathological process once triggered, thus the outcomes are inconclusive and remain as long as these therapies are administered. For this reason, orthopedic research investigates chondroprotective treatments able to reduce the local inflammatory microenvironment and to favor damaged articular cartilage anabolic activity.

Pulsed electromagnetic field (PEMF) stimulation has been already studied and proposed for the regeneration of musculoskeletal tissues such as cartilage [5], bone [6, 7], tendon [8] and ligament [9]. As far as cartilage tissue is concerned, PEMFs positively affect chondrocyte proliferation and ECM component synthesis [10]. However, the existing studies, performed with different PEMF physical parameters and exposure times, gave contradictory results [11-15], in terms of chondrocyte viability, DNA content, glycosaminoglycans (GAGs), Coll II, PGs and aggrecan gene expression and production. In a previous in vitro study, on bovine cartilage explants, we conducted a doseresponse study to find the optimal dosage, in terms of PEMF frequency and exposure intensity, able to stimulate significantly PG synthesis [16]. Furthermore, the stimulatory effect of PEMFs on PG synthesis in presence of IL1 $\beta$ and in combination with insulin-like growth factor 1 (IGF-I) was observed [17, 18]. PEMF and IGF-I showed an additive effect on PG synthesis in human OA cartilage explants cultured in the absence or presence of IL1 $\beta$ [19]. In in vivo studies, PEMF stimulation was able to limit the progression of OA of increasing severity [20-22], to reduce IL1 $\beta$ and tumor necrosis factor- $\alpha$ (TNF- $\alpha$ ) concentrations and to increase transforming growth factor- $\beta 1$ (TGF $\beta 1$ ) in the synovial fluid of sheep treated with autologous osteochondral grafts [23]. In clinical studies, PEMF stimulation was successfully used to treat pain, knee swelling and functionality, after total knee arthroplasty [24, 25] and in early knee OA patients [26]. In other studies, beneficial symptomatic effects $[27,28]$ or additional effects to physical treatments [29] were not observed in OA patients.

Even if PEMFs act on the joint microenvironment and have anabolic and chondroprotective actions, the mechanisms by which PEMFs exert their effects on biological systems remain not completely understood. To our knowledge, no histological and histomorphometric evaluations have assessed the effects of PEMF stimulation in an inflammatory $\mathrm{OA}$ microenvironment obtained in vitro with the addition of a high IL1 $\beta$ dose.

The present study aimed to analyze whether PEMF stimulation $(75 \mathrm{~Hz}, 1.5 \mathrm{mT})$, applied to bovine cartilage explants, derived from two different joints of the same animals, was able to counteract the catabolic effect of a high dose of IL1 $\beta$. In comparison to $2 \mathrm{D}$ monolayer chondrocyte cultures, the cartilaginous 3D explants cultured with IL1 $\beta$ are a well-accepted method to measure and evaluate the effect and mechanism of action of a therapy, by simultaneously evaluating chondrocytes and cartilage matrix and by closely mimicking the clinical situation of OA joint, in which a high dose of IL1 $\beta$ is found in the early stages of OA [30] and its concentration can be further increased by surgical intervention [2].

It was hypothesized that the PEMF stimulation alone, without the addition of other biological stimuli, might improve the production of the most important cartilage ECM components (PGs, GAGs, COLL II) and anabolic factor (TGF- $\beta 1$ ) and preserve cartilage structure, thus controlling OA development. To demonstrate this hypothesis it was adopted an already set and validated in vitro model of OA $[18,31]$, which used a high dose of IL1 $\beta$ (50 $\mathrm{ng} / \mathrm{ml}$ ) to create an inflammatory OA microenvironment. After $24 \mathrm{~h}, 7$ and 21 days of culture, PEMF effect, in combination or not with IL1 $\beta$, was evaluated through histological, histomorphometric and immunohistochemical analyses. In this study it was observed that PEMFs were able to counteract the progression of OA acting on both cartilage cellularity and ECM in cartilage previously treated with IL1 $\beta$.

\section{Methods}

\section{Cartilage explant cultures and treatment conditions}

Full-thickness explants of bovine articular cartilage were aseptically dissected, by using a $4 \mathrm{~mm}$ dermal punch (Stiefel Laboratories, Milan, Italy), from the metacarpophalangeal(MC) and metatarsophalangeal (MT) joints of 14-18-month-old animals (Limousine breed), as previously described [16-18]. The abattoir gave permission for the use of cartilage explants in this study and no ethical approval, from a recognized ethics committee, was required for this study. The cartilage was explanted shortly after the bovine slaughter and cartilage discs (three discs in each well) were cultured in $0.5 \mathrm{ml}$ culture medium in multiwells (Nunc, Denmark, $1.6 \mathrm{~cm}$ the diameter of each well). Before PEMF exposure and IL1 $\beta$ administration, all explants were allowed to equilibrate in culture for $48 \mathrm{~h}$ in DMEM/F12 (Life Technologies, Monza, Italy) supplemented with $10 \%$ FBS and antibiotics (penicillin 100 units $/ \mathrm{ml}$, streptomycin $0.1 \mathrm{mg} / \mathrm{ml}$ ) (complete medium) and for an additional $48 \mathrm{~h}$ in medium without serum, at $37{ }^{\circ} \mathrm{C}$ in an atmosphere of $5 \% \mathrm{CO}_{2}$. During the experiments, explants were cultured in complete medium in the absence and presence of IL1 $\beta(50 \mathrm{ng} / \mathrm{ml})$ [18]. Half cultures were exposed to PEMF throughout the entire culture period $(24 \mathrm{~h}, 7$ and 21 days). Medium was changed at the beginning of the exposure (time 0 ) and every 3 days. 
For each experimental time, 4 experimental groups were set up and each group was composed of 3 explant discs from MC and 3 more from MT joints:

- Control group (CTR): cartilage explants;

- IL1 $\beta$ group: cartilage explants submitted to IL1 $\beta$ administration;

- PEMF group: cartilage explants exposed to PEMFs;

- IL1 $\beta$ + PEMF group: cartilage explants submitted to IL1 $\beta$ and exposed to PEMFs.

\section{Characteristics of PEMFs and exposure conditions}

The PEMF generator system was the same as that used in previous studies [9, 16-18, 32, 33]. It consisted of a pair of circular Helmoltz coils of copper wire placed opposite to each other and in a signal generator (Igea S.p.A., Carpi, Italy). The multiwell plates were placed between this pair of Helmoltz coils, so that the plane of the coils was perpendicular to the multiwell plates, and the direction of the induced electric field was perpendicular to the direction of the magnetic field. The power generator produced a pulsed signal with the following parameters: the pulse duration was $1.3 \mathrm{~ms}$ and the frequency was $75 \mathrm{~Hz}$, yielding a duty cycle of $1 / 10$. The intensity peak of the magnetic field was $1.5 \mathrm{mT}$ and the induced electric field, as detected with a standard coil probe (50 turns, $0.5 \mathrm{~cm}$ internal diameter of the coil probe, 0.2 copper diameter), was $0.07 \mathrm{mV} / \mathrm{cm}$.

The intensity of the magnetic field was detected between two coils from one side to the other, by the Hall probe of the Gaussmeter (LE, Gaussmeter DG500, USA), with a reading sensitivity of $0.2 \%$. Inside this area, where the multiwell plate was placed, the magnetic field was uniform. Cartilage explants were exposed to continuous PEMF for $24 \mathrm{~h}, 7$ or 21 days. PEMF unexposed cultures were placed inside the same incubator at a distance where no difference from background magnetic field was observed when the PEMF generator was turned on. In each experiment, controls and treatments were performed in triplicate wells.

\section{Histology}

At the end of the experimental times, cartilage explants, cultured in the above mentioned conditions, were routinely processed for paraffin embedding procedures. Briefly, samples were fixed for $24 \mathrm{~h}$ in $10 \%$ neutral buffered formalin solution in PBS, extensively rinsed in distilled water, dehydrated in graded alcohol solutions (70, $95 \%$, two times and $100 \%$, three times, one hour for each step), cleared in xylene e finally paraffin embedded. Sections $(5 \pm 1 \mu \mathrm{m})$ were cut along the longitudinal axis of the samples by a semi-automated microtome (Microm H340E, Germany) and stained with Toluidine Blue, for the PG quantification and Safranin O-Fast green staining, for the GAG quantification. Three non-consecutive sections for each sample were evaluated, by 2 independent histologists through a semi-quantitative score (modified O'Driscoll score) [34]. The modified O'Driscoll score (Table 1: minimum $0=$ osteoarthritic cartilage $-\max -$ imum $11=$ normal intact cartilage) analyzed 4 cartilage aspects: Safranin O staining, surface regularity, cellularity and chondrocyte clustering.

\section{Immunohistochemical stainings (TGF $\beta 1$ and Coll II)}

Cartilage sections were dewaxed in decreasing graded ethanol solutions until PBS rinsing for $20 \mathrm{~min}$ and then immunostained for TGF $\beta 1$ and Coll II. Briefly, after fixation, sections were extensively rinsed in PBS and permeabilized by incubation in $0.3 \%$ hydrogen peroxide in PBS solution for 15 min. For Coll II immunostaining, sections were pre-treated for antigen unmasking with $0.2 \%$ Pronase (P-8811, Sigma, Mo, USA) solution in PBS for $30 \mathrm{~min}$ at $37^{\circ} \mathrm{C}$. Then, $10 \%$ normal serum was added for $1 \mathrm{~h}$ at room temperature to block nonspecific antibody binding and the primary antibodies (rabbit polyclonal antibody anti TGF $\beta 1$, sc-146 and mouse monoclonal antibody anti collagen II, sc-52658, 1:50 dilutions, Santa Cruz Biotechnology, CA, USA) were applied and incubated overnight at $4{ }^{\circ} \mathrm{C}$. After rinsing in PBS, slides were incubated with appropriate biotinylated secondary antibody and with horseradish peroxidasestreptavidin complex for $1 \mathrm{~h}$ each (ABC Staining System, Biotechnology, CA, USA). Sample reaction was developed with 3,3-diaminobenzidine substrate and permanently mounted. Negative controls, by omitting the primary antibody, were included to check proper specificity and performance of the applied reagents.

Table 1 Modified O'Driscoll score

\begin{tabular}{lll}
\hline Parameter & Feature & Grade \\
\hline Safranin O staining & Normal or nearly normal & 3 \\
& Moderate & 2 \\
& Slight & 1 \\
& None & 0 \\
Surface regularity & Smooth and intact & 3 \\
& Superficial horizontal lamination & 2 \\
& Fissures 25-100 \% of the thickness & 1 \\
& Severe disruption, including fibrillation & 0 \\
Cellularity & Normal cellularity & 3 \\
& Slight hypocellularity & 2 \\
& Moderate hypocellularity & 1 \\
& Severe hypocellularity & 0 \\
& No clusters & 2 \\
& $<25 \%$ of the cells & 1 \\
& $25-100 \%$ of the cells & 0 \\
\hline
\end{tabular}




\section{Histomorphometric measurements}

Slices stained with Toluidine Blue or Safranin O-Fast Green and immunostained for TGF $\beta 1$ or Coll II were observed by a light microscope (BX51, Olympus Italia Srl, Segrate-Milano, Italy) and three Regions of Interest (ROI) for each slide were grabbed at a $40 \times$ magnification. Image analysis by Leica Q-Win Software (Leica Microsystem, Wetzlar, Germany) was performed and the threshold applied was One-dimensional (LUTs: Lookup Table Transforms) based on selected ranges of RGB channels to measure the following parameters expressed as percentage:

- GAGs, as the ratio between the Safranin O stained areas and the total ROI areas;

- PGs, as the ratio between the Toluidine Blue stained areas and the total ROI areas;

- TGF $\beta 1$, as the ratio between the immunopositive stained areas and the total ROI areas;

- Coll II, as the ratio between the immunopositive stained areas and the total ROI areas.

\section{Statistical analysis}

Statistical analysis was performed using the IBM SPSS Statistics v.21.0 software (IBM Corp, USA). Firstly, normal distribution (Kolmogroc-Smirnov test) and homoscedasticity (Levene test) of data were verified, then, the non-parametric Kruskal Wallis test followed by MannWhiteny $U$ test for pairwise comparisons were performed. The $P$-value was adjusted according to the Dunn-Sidak correction [adj $p=1-(1-p)^{\mathrm{k}}$, where $k$ is the number of pairwise comparisons]. Since the preliminary Kruskal-Wallis test by distinguish MC and MP joints did not show significant differences between the two harvesting sites within each type of treatment, data were analyzed when merged.

\section{Results}

The in vitro cartilage OA model after IL1 $\beta$ administration In the CTR Group the production of PGs, GAGs and COLL II significantly decreased at 7 days in comparison with cultures at $24 \mathrm{~h}$ by $-47 \%(p=0.022),-58 \%(p=$ $0.008)$ and $-27 \%(p=0.038)$, respectively (Figs. $1,2,3)$.

As expected, the treatment with IL1 $\beta$ significantly decreased the PGs, GAGs, Coll II and TGF $\beta 1$ production in cartilage explants as compared to CTR at each experimental time, except for TGF $\beta 1$ at 21 days (Figs. 1, 2, 3, 4). Specifically, PGs decreased by 96$99 \%$ (at $24 \mathrm{~h} p=0.0005$, at 7 days $p=0.0005$ and at 21 days $p=0.0005)$, GAGs by $88 \%$ at $24 \mathrm{~h}(p=0.0005)$ and about $73 \%$ at $7(p=0.008)$ and $21(p=0.03)$ days and Coll II by $92-96 \%$ (at $24 \mathrm{~h} p=0.0005$, at 7 days $p=$ 0.0005 and at 21 days $p=0.04$ ) (Figs. $1,2,3)$. The production of TGF $\beta 1$ significantly decreased at $24 \mathrm{~h}(p=0.0005$,

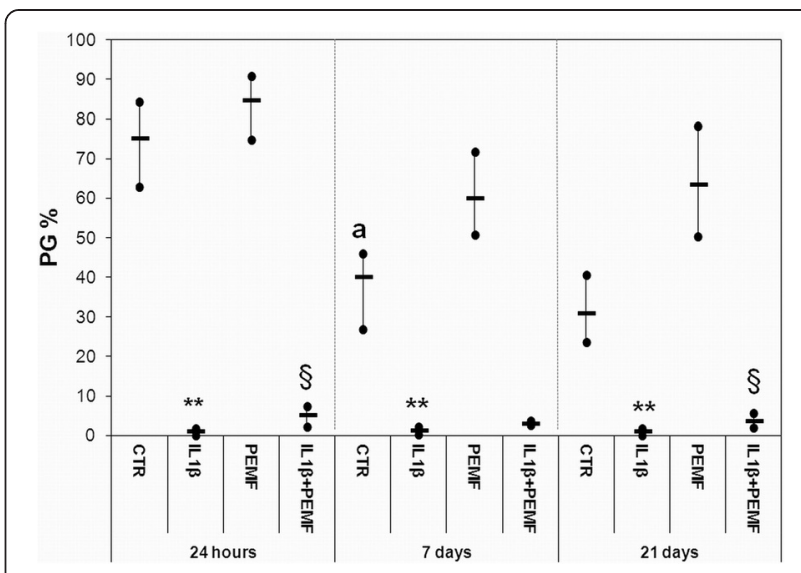

Fig. 1 Boxplots of histomorphometric measurements of PGs expressed as percentage in bovine cartilage explants. Black line = median; extreme values $=$ minimum-maximum. Control (CTR), exposed to PEMF (PEMF), treated with IL1 $\beta$ (IL1 $\beta$ ) or treated with IL1 $\beta$ and stimulated with PEMF $($ IL $1 \beta+$ PEMF) explants. $24 \mathrm{~h}, 7$ and 21 days $(n=6)$. Mann-Whitney $U$ test: - Within each experimental time: IL1 $\beta$ versus CTR (**, $p<0.005) ; \| L 1 \beta+$ PEMF versus IL1 $\beta(\S, p<0.05)$; - Within each group: 7 days versus $24 \mathrm{~h}$ $\left({ }^{a}, p<0.05\right)$

$47 \%)$ and 7 days ( $p=0.017,68 \%$ ) (Fig. 4). Figure 5 shows the immunohistochemical and histological images of explants stained for Coll II, TGF $\beta 1$ and Safranin O at $24 \mathrm{~h}$ and 21 days.

Because of the IL1 $\beta$ effect, the O'Driscoll score progressively worsened by 50-60\%, during the experimental times, in comparison to CTR (at $24 \mathrm{~h} p=0.0005$, at 7 days $p=0.0005$ and at 21 days $p=0.04$ ) (Fig. 6). By analyzing each score parameter (Table 2), it was noted

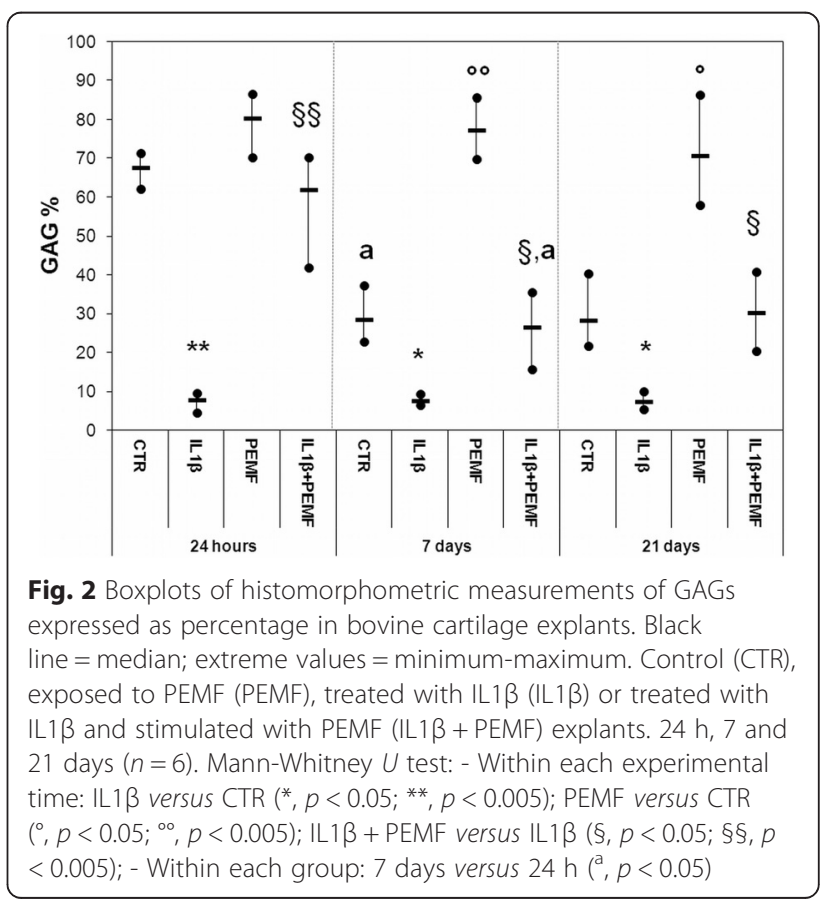




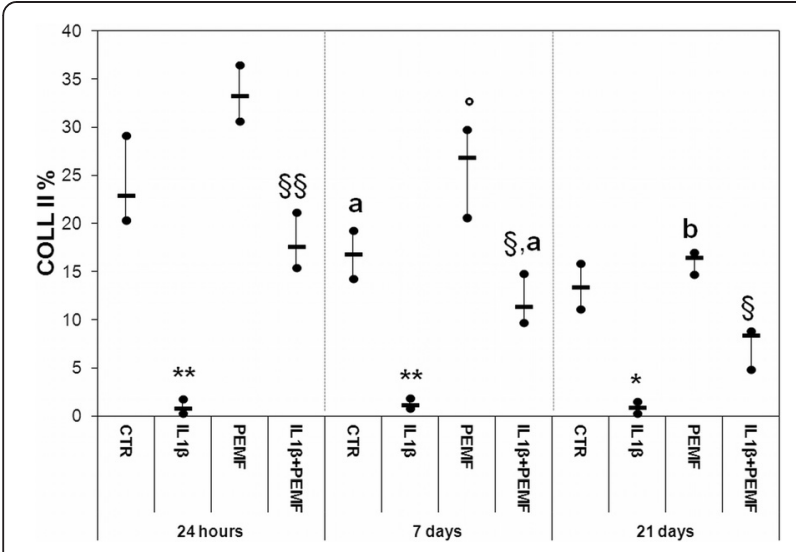

Fig. 3 Boxplots of histomorphometric measurements of Coll ॥ expressed as percentage in bovine cartilage explants. Black line = median; extreme values = minimum-maximum. Control (CTR), exposed to PEMF (PEMF), treated with IL1 $\beta(\mathrm{L} 1 \beta)$ or treated with IL1 $\beta$ and stimulated with PEMF (IL1 $\beta+$ PEMF) explants. 24 h, 7 and 21 days $(n=6)$. Mann-Whitney $U$ test: - Within each experimental time: IL1 $\beta$ versus CTR $\left({ }^{*}, p<0.05 ;{ }^{* *}, p<0.005\right)$; PEMF versus CTR $\left({ }^{\circ}, p<0.05\right)$; IL1 $\beta$ + PEMF versus IL1 $\beta$ (§, $p<0.05 ; \S \S, p<0.005)$; - Within each group: 7 days versus $24 \mathrm{~h}\left({ }^{\mathrm{a}}, p<0.05\right) ; 21$ days versus 7 days $\left({ }^{\mathrm{b}}, p<0.005\right)$

that, the cellularity $(p=0.025)$ and chondrocyte clustering $(p=0.025)$ decreased significantly by $50 \%$ in the CTR group from 7 to 21 days and from 24 h to 7 days, respectively. At $24 \mathrm{~h}$, the reduction induced by IL1 $\beta$ on each O'Driscoll score parameter in comparison with CTR was $50 \%$ for Safranin-O staining $(p=0.0005)$, surface regularity $(p=0.002)$ and cellularity $(p=0.0005)$ and $75 \%$ for chondrocyte clustering $(p=0.0005)$, whereas at 7 and 21 days it was $50 \%$ for surface regularity (at 7 days $p=0.0005$ and at 21 days $p=0.0005), 50-100 \%$ for

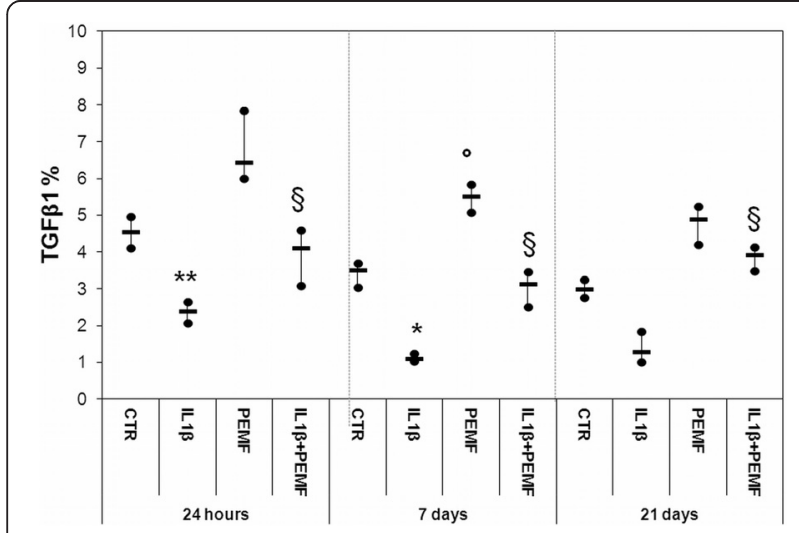

Fig. 4 Boxplots of histomorphometric measurements of TGF- $\beta 1$ expressed as percentage in bovine cartilage explants. Black line = median; extreme values = minimum-maximum. Control (CTR), exposed to PEMF (PEMF), treated with IL1 $\beta$ (IL1 $\beta$ ) or treated with IL1 $\beta$ and stimulated with PEMF (IL1 $\beta+$ PEMF) explants. 24 h, 7 and 21 days $(n=6)$. Mann-Whitney $U$ test: - Within each experimental time: IL $1 \beta$ versus CTR (*, $p<0.05$; $\left.{ }^{* *}, p<0.005\right)$; PEMF versus CTR $\left({ }^{\circ}, p<0.05\right) ; \mathrm{IL} 1 \beta+$ PEMF versus IL $1 \beta(\S, p<0.05)$ cellularity (at 7 days $p=0.001$ and at 21 days $p=0.001$ ) and $100 \%$ for chondrocyte clustering (at 7 days $p=$ 0.001 and at 21 days $p=0.02$ ).

\section{Effects of PEMF stimulation}

As also observed by histology in Fig. 5, in bovine cartilage explants stimulated by PEMF, no significant increases in PGs were found in comparison with CTR explants at each experimental time (Fig. 1). Conversely, PEMF stimulated GAG production at $7(p=0.0005,170 \%)$ and $21(p=$ $0.005,151 \%)$ days, as well as Coll II $(p=0.005,59 \%)$ and TGF $\beta 1(p=0.005,58 \%)$ at 7 days (Figs. $2,3,4)$. By analyzing data over time, Coll II in the PEMF group diminished from 7 to 21 days $(p=0.004,38 \%)$.

When bovine cartilage explants were cultured with IL1 $\beta$ and were stimulated with PEMFs, very high increases in PG production compared to the IL1 $\beta$ group were observed at $24 \mathrm{~h}(p=0.01,439 \%)$ and 21 days $(p=0.04$, 235 \%) (Fig. 1). The IL1 $\beta$ + PEMF group also showed increases in GAG production in comparison to the IL1 $\beta$ group at $24 \mathrm{~h}(p=0.0005,684 \%), 7(p=0.02,250 \%)$ and 21 days $(p=0.01,307 \%)$ (Fig. 2$)$. Coll II increased massively in the IL $1 \beta+$ PEMF group at $24 \mathrm{~h} \quad(p=0.002$, $1890 \%), 7(p=0.02,835 \%)$ and 21 days $(p=0.007$, $734 \%$ ) in comparison to IL1 $\beta$ (Fig. 3). GAG and Coll II synthesis decreased by $57 \%(p=0.02)$ and $35 \%(p=$ $0.02)$, respectively, from $24 \mathrm{~h}$ to 7 days in the IL $1 \beta+$ PEMF group. Regarding TGF $\beta 1$ production, it increased progressively at all experimental times (at $24 \mathrm{~h}$ $p=0.005,71 \%$, at 7 days $p=0.02,184 \%$ and at 21 days $p=0.007,204 \%)$ in comparison to the IL1 $\beta$ group (Fig. 4).

The O'Driscoll score results of the PEMF-treated group did not differ from those of the CTR group at each experimental time (Fig. 6). Taking into account each O'Driscoll score parameter (Table 2), PEMF stimulation improved chondrocyte clustering at 7 days $(p=0.0005,100 \%)$ and Safranin-O staining at $7(p=0.0005,100 \%)$ and 21 days ( $p=0.03,200 \%$ ) compared to that of the CTR group.

The effect of PEMF stimulation, applied to IL1 $\beta$-treated cartilage explants (IL1 $\beta+$ PEMF group), improved the O'Driscoll score in comparison to the IL1 $\beta$ group, at both $24 \mathrm{~h}(p=0.02,75 \%)$ and 21 days $(p=0.03,150 \%)$ (Fig. 6). A decrease in O'Driscoll score was highlighted between $24 \mathrm{~h}$ and 7 days in the IL1 $\beta+$ PEMF group $(p=0.007$, $43 \%)$. The IL1 $\beta+$ PEMF group improved by about $100 \%$ for Safranin-O staining $(p=0.02)$ and cellularity $(p=$ $0.003)$ at $24 \mathrm{~h}$, for chondrocyte clustering $(p=0.008)$ and Safranin-O staining $(p=0.008)$ at 7 days, and for surface regularity $(p=0.003)$, Safranin-O staining $(p=0.001)$, chondrocyte clustering $(p=0.005)$ and cellularity $(p=$ 0.001 ) at 21 days compared to those of the IL1 $\beta$ group (Table 2). In addition, surface regularity of the IL1 $\beta+$ 


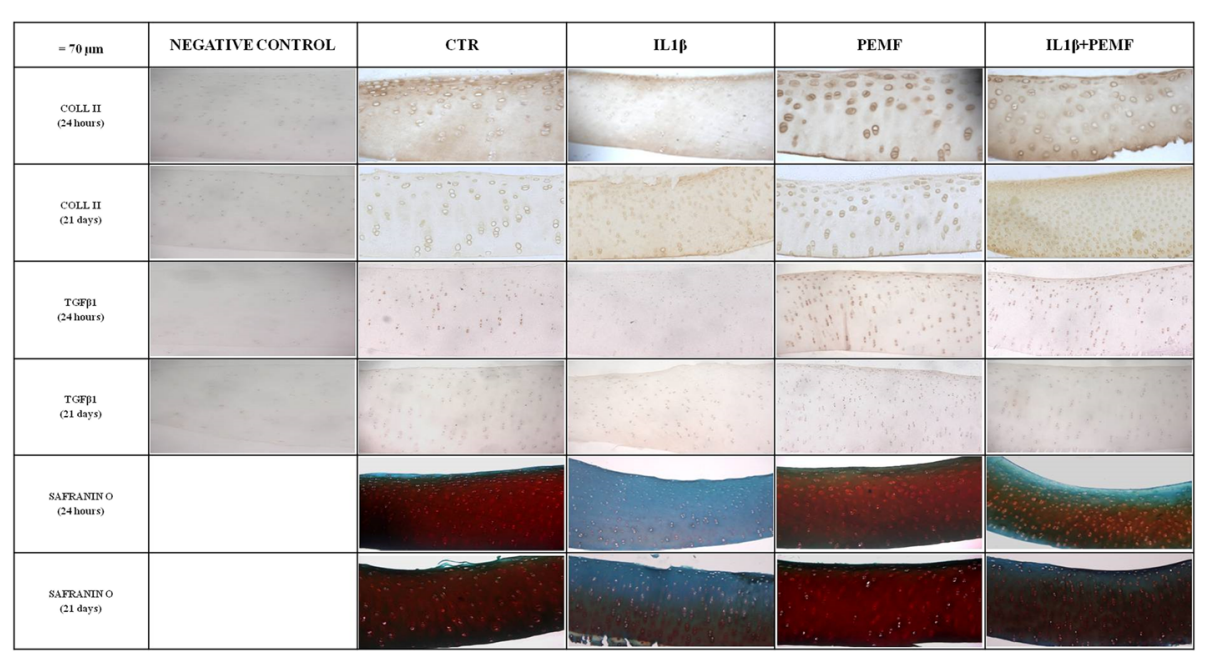

Fig. 5 Immunohistochemical and histological images of bovine cartilage explants. Control (CTR), exposed to PEMF (PEMF), treated with IL1 $\beta$ (IL1 $\beta)$ or treated with IL1 $\beta$ and stimulated with PEMF (IL1 $\beta+$ PEMF) explants. $24 \mathrm{~h}$ and 21 days. Magnification at 20x. Bar $=70 \mu \mathrm{m}$. Negative control column contains 4 images of the negative control of the relative markers (COLL II and TGF $\beta 1$ )

PEMF group decreased by $50 \%(p=0.003)$ from 24 h to 7 days (Table 2 ).

\section{Discussion}

The present study aimed to evaluate the effects and mechanisms of action of PEMF stimulation $(75 \mathrm{~Hz}, 1.5 \mathrm{mT}$ ) in preserving cartilage from OA structural deterioration. The in vitro effects were investigated on bovine cartilage explants harvested from two different joints. To simulate the OA microenvironment, a high IL1 $\beta$ concentration (50 ng/ $\mathrm{ml}$ ) was administered in explant cultures, as set in an our previous in vitro study [30], for a longer period of 21 days

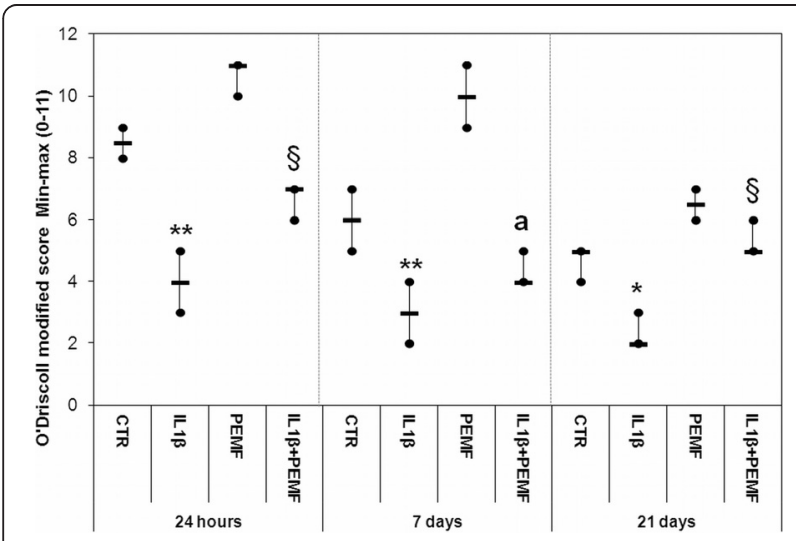

Fig. 6 Boxplots of total O'Driscoll modified score in bovine cartilage explants. Black line = median; extreme values = minimum-maximum. Control (CTR), exposed to PEMF (PEMF), treated with IL1 $\beta$ (IL1 $\beta$ ) or treated with $\mathrm{IL} 1 \beta$ and stimulated with PEMF (IL1 $\beta+$ PEMF) explants. 24 h, 7 and 21 days $(n=6)$. Mann-Whitney $U$ test: - Within each experimental time: IL1 $\beta$ versus CTR ( $\left.{ }^{*}, p<0.05 ;{ }^{* *}, p<0.005\right)$; IL1 $\beta+$ PEMF versus IL $1 \beta(\S, p<0.05)$; - Within each group: 7 days versus $24 \mathrm{~h}\left({ }^{\mathrm{a}}, \mathrm{p}<0.05\right)$ and the changes in the structure and cellularity of CTR and IL1 $\beta$-treated cartilage explants, either exposed or not to PEMFs, were analyzed. The concentration of IL-1 $\beta$ found in the synovial fluid of patients affected by $\mathrm{OA}$ is between 0.068 and $0.33 \mathrm{pg} / \mathrm{ml}$ [35]. However, most of the in vitro literature studies, that aimed to re-create an inflammatory microenvironment, added $10 \mathrm{ng} / \mathrm{ml}$ of IL- $1 \beta$ in the medium of cartilage explants [36-38], while there are other authors that employed 50 or $100 \mathrm{ng} / \mathrm{ml}$ [39-41]. The so high concentration of IL-1 $\beta$ used in the present study was chosen to employed an intermediate cytokine dosage and to evaluate if PEMF stimulation is able to restore cartilage ECM biological and morphological properties also with a huge concentration of IL-1 $\beta$ than the most employed one $(10 \mathrm{ng} / \mathrm{ml})$. The current data confirmed the hypotheses of the study. The addition of a high dose of IL1 $\beta$ to the culture medium induced a significant reduction, not only in structural parameters, but also ECM components and TGF $\beta 1$ synthesis, a decline observed at all experimental times until 21 days. These results are in agreement with those of previous in vitro studies, that observed GAGs, PGs and Coll II loss with the addition of IL1 $\beta$ in the culture medium of human, bovine and horse cartilage explants [30, 42, 43]. Although the joint inflammatory microenvironment of OA consists of different inflammatory mediators, such as matrix metalloproteinases (MMPs), the disintegrin and metalloproteinases with thrombospondin motifs (ADAMTs) and other pro-inflammatory cytokines, TNF- $\alpha$ and Interleukin-6 (IL$6)$, data suggested that IL1 $\beta$ might have the strongest effect. Indeed, with the addition of IL1 $\beta$ in the culture medium, the most important components of cartilage ECM and TGF $\beta 1$ synthesis were reduced and other cartilage structural parameters (matrix and cellularity) were 
Table 2 Median values (minimum and maximum values) of O'Driscoll modified score parameters in cartilage explants

\begin{tabular}{|c|c|c|c|c|c|}
\hline Experimental time & O'driscoll score parameters & CTR & IL1 $\beta$ & PEMF & IL1 $\beta+$ PEMF \\
\hline \multirow[t]{4}{*}{$24 \mathrm{~h}$} & Safranin-O staining & $2(2-3)$ & $1(1-2)^{* *}$ & $3(2-3)$ & $2(2-2)^{\S}$ \\
\hline & Surface regularity & $2(2-3)$ & $1(1-2)^{* *}$ & $3(3-3)$ & $2(2-2)$ \\
\hline & Chondrocyte clustering & $2(1-2)$ & $0.5(0-1)^{* *}$ & $2(2-2)$ & $1(1-2)$ \\
\hline & Cellularity & $2(2-3)$ & $1(1-1)^{* *}$ & $3(2-3)$ & $2(1-2)^{\S \S}$ \\
\hline \multirow[t]{4}{*}{7 days } & Safranin-O staining & $1(1-2)$ & $0(0-1)$ & $3(2-3)^{\circ}$ & $1(1-2)^{\S}$ \\
\hline & Surface regularity & $2(1-3)$ & $1(0-1)^{* *}$ & $3(2-3)$ & $1(1-2)$ \\
\hline & Chondrocyte clustering & $1(1-1)$ & $0(0-1)^{* * a}$ & $2(1-2)^{\circ}$ & $1(1-2)^{\S}$ \\
\hline & Cellularity & $2(1-2)$ & $1(0-1)^{* *}$ & $2(2-3)$ & $1(1-2)$ \\
\hline \multirow[t]{4}{*}{21 days } & Safranin-O staining & $1(1-2)$ & $0(0-1)$ & $2(2-2)^{\circ}$ & $1(1-1)^{\S \S}$ \\
\hline & Surface regularity & $2(1-2)$ & $1(1-1)^{* *}$ & $2(2-2)$ & $2(1-2)^{\S \S, b}$ \\
\hline & Chondrocyte clustering & $1(1-1)$ & $0(0-0)^{*}$ & $1(1-1)^{\mathrm{b}}$ & $1(1-2)^{\S}$ \\
\hline & Cellularity & $1(1-1)$ & $0(0-1)^{* *, a}$ & $1.5(1-2)$ & $1(1-1)^{\S \S}$ \\
\hline
\end{tabular}

Control (CTR), exposed to PEMF (PEMF), treated with IL1 $\beta$ (IL1 $\beta$ ) or treated with IL1 $\beta$ and stimulated with PEMF (IL1 $\beta+$ PEMF) explants. $24 \mathrm{~h}, 7$ and 21 days $(n=6)$ Mann-Whitney $U$ test: - Within each experimental time: IL $1 \beta$ versus CTR $\left({ }^{*}, p<0.05 ;{ }^{*}, p<0.005\right)$; PEMF versus $\left(\mathrm{CTR}\left({ }^{\circ}, p<0.05 ;{ }^{\circ}, p<0.005\right) ;\right.$ IL1 $\beta+$ PEMF versus IL $1 \beta$ $(\S, p<0.05 ; \S \S, p<0.005)$; Within each group: 7 days versus $24 \mathrm{~h}\left({ }^{\mathrm{a}}, p<0.05\right) ; 21$ days versus 7 days $\left({ }^{\mathrm{a}}, p<0.05 ;{ }^{\mathrm{b}}, p<0.005\right)$

compromised. Coll II, PGs and GAGs are the most abundant components of cartilage ECM, which are responsible for the ECM organization and give shear and tensile properties and the ability to resist compressive loads to cartilage.

Secondly, the stimulation with PEMFs, applied to healthy explants, did not modify PG content or the structural parameters at any of the experimental times, and moreover, increases in GAGs and Coll II were observed after $24 \mathrm{~h}$ of culture. Instead, PEMFs improved all the analyzed ECM proteins and the structural parameters in IL1 $\beta$-treated explants, also at 21 days of culture. Similar results were published by Brighton et al., in 2006 and 2008 using capacitively coupled electric fields (CCEF). They observed an increase in Coll II and PG production in human and bovine cartilage explants, treated or not with IL1 $\beta$, after stimulation $[44,45]$. Finally, in this study it was also observed that IL1 $\beta$ and PEMFs have an opposite effect on TGF $\beta 1$, the anabolic growth factor that induces matrix production, mesenchymal stem cell chondrogenic differentiation and chondrocyte proliferation [46].

This study allowed a more complete view of the role of PEMFs that has been already investigated in previous our in vitro studies $[18,31]$. The strengths of the study were: 1) the histological and histomorphometric evaluations on in vitro cartilage explants, stimulated with PEMF parameters already used in patients suffering from knee pain due to cartilage degeneration [47]; 2) the long-term culture of cartilaginous explants (21 days) and 3) the comparative analysis in two different joints. A limitation of the study was the absence, in the culture explants model, of the subchondral bone, that is recognized to play a role in OA development. It is noteworthy that the score employed in this study is a modification of the score proposed by O'Driscoll SW, et al., in 1988 [34]: only the parameters that regard the matrix, the structure and the chondrocyte hypocellularity and clustering could be evaluated.

In comparison to $2 \mathrm{D}$ monolayer chondrocyte cultures and in vivo situation, the 3D cartilage explants, adopted in this study, are a more homogenous model to evaluate cartilage metabolism. Chondrocytes are maintained in their physiological microenvironment, the de-differentiation phenomenon is prevented and the inter-individual variables are reduced [48]. In addition, the use of cartilage explants is a more suitable in vitro method than chondrocytes embedded in alginate cultures to study PEMF and IL1 $\beta$ effects, because the chondrocytes are embedded in their native ECM. In fact the changes in ECM composition may play an important role in maintaining osteoarthritis damage and the alterations in hyaluronate degradation can be an alternative mechanism for the regulation of proteoglycan release from cartilage following IL1 $\beta$ stimulation [49]. 3D explants are reliable even at long experimental times because the effects of IL1 $\beta$ treatment and PEMF stimulation were evident also at 21 days of culture. Many in vitro culture studies evaluated bovine cartilage because of the advantageous easy availability and similar thickness to human cartilage [50]. Moreover, the metabolic activity and structural composition of bovine cartilage is reported to be quite similar to those of humans [50].

\section{Conclusions}

This study shows that the addition of IL1 $\beta$ to the culture medium has a detrimental effect on cartilage explants by reducing PG, GAG, Coll II and TGF $\beta 1$ quantification and 
O'Driscoll modified score parameters. PEMF stimulation is able to counteract these catabolic effects both on ECM and chondrocyte features. It was also found that PEMFs have a greater effect on cartilage explants compromised by IL1 $\beta$ in comparison to normal tissues. These results confirm the role of PEMFs in chondroprotection and, as shown by the long-term results, they suggest their ability to limit OA progression.

\section{Abbreviations}

OA: Osteoarthritis; PEMF: Pulsed electromagnetic field; ECM: Extracellular matrix; IL1 $\beta$ : Interleukin 1 $\beta$; PGs: Proteoglycans; Coll II: Collagen type II; NSAIDs: Nonsteroidal anti-inflammatory drugs; HA: Hyaluronic acid; GAGs: Glycosaminoglycans; IGF-I: Insulin-like growth factor 1; TNF-a: Tumor necrosis factor- $\alpha$; TGF $\beta 1$ : Transforming growth factor- $\beta 1$;

MC: Metacarpophalangeal; MT: Metatarsophalangeal; CTR: Control group; MMPs: Metalloproteinases; ADAMTs: The disintegrin and metalloproteinases with thrombospondin motifs; IL-6: Interleukin-6; CCEF: Capacitively coupled electric fields.

\section{Competing interests}

The authors declare that they have no competing interests. Stefania Setti is employed in IGEA, that developed the PEMF generator.

\section{Authors' contributions}

FV has been involved in acquisition, analysis and interpretation of data. MF has made substantial contributions to conception and design of the study and she has been involved in revising the manuscript critically for important intellectual content. GG has made substantial contributions to analysis and interpretation of data and carried out statistical analysis. AO has made substantial contributions to conception and design of the study and was involved in the in vitro culture of cartilage explants. MDM has made substantial contributions to conception and design of the study and revised the manuscript. AP has made substantial contributions to acquisition and analysis of data. SS provided the PEMF generator and has made substantial contributions to analysis and interpretation of data. MT has made substantial contributions to analysis and interpretation of data and has been involved in revising the manuscript critically for important intellectual content. All authors have been involved in drafting the manuscript, have given final approval of the version to be published and agree to be accountable for all aspects of the work in ensuring that questions related to the accuracy or integrity of any part of the work are appropriately investigated and resolved.

\section{Acknowledgements}

Funding for the study was provided by "Pathogenesis and molecular targets in degenerative musculoskeletal diseases" project FIRB No. RBAP10KCNS - 2010 and was supported partly by grants from Programma di Ricerca Regione Emilia Romagna-Università 2010-2012 to MT (Biological and Biophysical Stimulation on Implant Osteolysis and Aseptic Loosening Conditions: Effects of Pulsed Electromagnetic Fields and Platelet Derivatives Project).

\section{Author details}

'Department Rizzoli RIT, Rizzoli Orthopedic Institute, Laboratory of Biocompatibility, Innovative Technologies and Advanced Therapies, Bologna 40136, Italy. ${ }^{2}$ Laboratory of Preclinical and Surgical Studies, Rizzoli Orthopedic Institute, Bologna 40136, Italy. ${ }^{3}$ Department of Morphology, Surgery and Experimental Medicine, University of Ferrara, Ferrara 44121, Italy. ${ }^{4}$ IGEA Clinical Biophysic, Carpi (Modena) 41012, Italy.

Received: 25 July 2015 Accepted: 7 October 2015

Published online: 20 October 2015

\section{References}

1. Gao Y, Liu S, Huang J, Guo W, Chen J, Zhang L, et al. The ECM-cell interaction of cartilage extracellular matrix on chondrocytes. Biomed Res Int. 2014:2014:648459. doi:10.1155/2014/648459.

2. Fitzsimmons RJ, Gordon SL, Kronberg J, Ganey T, Pilla AA. A Pulsing Electric Field (PEF) Increases Human Chondrocyte Proliferation through a
Transduction Pathway Involving Nitric Oxide Signaling. J Orthop Res. 2008;26:854-9. doi:10.1002/jor.20590.

3. Braund R, Abbott JH. Recommending NSAIDs and paracetamol: a survey of New Zealand physiotherapists' knowledge and behaviours. Physiother Res Int. 2011;16:43-9. doi:10.1002/pri.472.

4. Karatosun V, Unver B, Ozden A, Ozay Z, Gunal I. Intra-articular hyaluronic acid compared to exercise therapy in osteoarthritis of the ankle. A prospective randomized trial with long-term follow-up. Clin Exp Rheumatol. 2008;26:288-94.

5. van Bergen CJ, Blankevoort L, de Haan RJ, Sierevelt IN, Meuffels DE, d'Hooghe PR, et al. Pulsed electromagnetic fields after arthroscopic treatment for osteochondral defects of the talus: double-blind randomized controlled multicenter trial. BMC Musculoskelet Disord. 2009:10:83. doi:10.1186/1471-2474-10-83.

6. Hannemann PF, van Wezenbeek MR, Kolkman KA, Twiss EL, Berghmans CH, Diven PA, et al. CT scan-evaluated outcome of pulsed electromagnetic fields in the treatment of acute scaphoid fractures: a randomised, multicentre, double-blind, placebo-controlled trial. Bone Joint J. 2014;96-B:1070-6. doi:10.1302/0301-620X.96B8.33767.

7. Ongaro A, Pellati A, Bagheri L, Fortini C, Setti S, De Mattei M. Pulsed electromagnetic fields stimulate osteogenic differentiation in human bone marrow and adipose tissue derived mesenchymal stem cells. Bioelectromagnetics. 2014;35:426-36. doi:10.1002/bem.21862. Epub 2014 Aug 6.

8. de Girolamo L, Viganò M, Galliera E, Stanco D, Setti S, Marazzi MG, et al. In vitro functional response of human tendon cells to different dosages of low-frequency pulsed electromagnetic field. Knee Surg Sports Traumatol Arthrosc. 2014. [Epub ahead of print].

9. Benazzo F, Zanon G, Pederzini L, Modonesi F, Cardile C, Falez F, et al. Effects of biophysical stimulation in patients undergoing arthroscopic reconstruction of anterior cruciate ligament: prospective, randomized and double blind study. Knee Surg Sports Traumatol Arthrosc. 2008;16:595-601. doi:10.1007/s00167-008-0519-9.

10. Chang SH, Hsiao YW, Lin HY. Low-frequency electromagnetic field exposure accelerates chondrocytic phenotype expression on chitosan substrate. Orthopedics. 2011:34:20. doi:10.3928/01477447-20101123-10.

11. Štolfa S, Škorvánek M, Štolfa P, Rosocha J, Vaško G, Sabo J. Effects of Static Magnetic Field and Pulsed Electromagnetic Field on Viability of Human Chondrocytes in vitro. Physiol Res. 2007;56 Suppl 1:S45-9.

12. Schmidt-Rohlfing B, Silny J, Woodruff S, Gavenis K. Effects of pulsed and sinusoid electromagnetic fields on human chondrocytes cultivated in a collagen matrix. Rheumatol Int. 2008;28:971-7. doi:10.1007/s00296-008-0565-0.

13. Chang CH, Loo ST, Liu HL, Fang HW, Lin HY. Can low frequency electromagnetic field help cartilage tissue engineering? J Biomed Mater Res. 2010;92A:843-51. doi:10.1002/jbm.a.32405.

14. Sadoghi P, Leithner A, Dorotka R, Vavken P. Effect of Pulsed Electromagnetic Fields on the Bioactivity of Human Osteoarthritic Chondrocytes. Orthopedics. 2013;36:e360-5. doi:10.3928/01477447-20130222-27.

15. Bobacz K, Graninger WB, Amoyo L, Smolen JS. Effect of pulsed electromagnetic fields on proteoglycan biosynthesis of articular cartilage is age dependent. Ann Rheum Dis. 2006;65:949-51.

16. De Mattei M, Fini M, Setti S, Ongaro A, Gemmati D, Stabellini G, et al. Proteoglycan synthesis in bovine articular cartilage explants exposed to different low-frequency low-energy pulsed electromagnetic fields. Osteoarthritis Cartilage. 2007;15:163-8.

17. De Mattei M, Pellati A, Pasello M, Ongaro A, Setti S, Massari L, et al. Effects of physical stimulation with electromagnetic field and insulin growth factor-l treatment on proteoglycan synthesis of bovine articular cartilage. Osteoarthritis Cartilage. 2004;12:793-800.

18. De Mattei M, Pasello M, Pellati A, Stabellini G, Massari L, Gemmati D, et al. Effects of electromagnetic fields on proteoglycan metabolism of bovine articular cartilage explants. Connect Tissue Res. 2003:44:154-9.

19. Ongaro A, Pellati A, Masieri FF, Caruso A, Setti S, Cadossi R, et al. Chondroprotective effects of pulsed electromagnetic fields on human cartilage explants. Bioelectromagnetics. 2011;32:543-51. doi:10.1002/bem.20663.

20. Veronesi F, Torricelli P, Giavaresi G, Sartori M, Cavani F, Setti S, et al. In vivo effect of two different pulsed electromagnetic field frequencies on osteoarthritis. J Orthop Res. 2014:32:677-85. doi:10.1002/jor.22584.

21. Fini M, Giavaresi G, Torricelli P, Cavani F, Setti S, Canè V, et al. Pulsed electromagnetic fields reduce knee osteoarthritic lesion progression in the aged Dunkin Hartley guinea pig. J Orthop Res. 2005;23:899-908.

22. Fini M, Torricelli P, Giavaresi G, Aldini NN, Cavani F, Setti S, et al. Effect of pulsed electromagnetic field stimulation on knee cartilage, subchondral 
and epyphiseal trabecular bone of aged Dunkin Hartley guinea pigs. Biomed Pharmacother. 2008;62:709-15.

23. Benazzo F, Cadossi M, Cavani F, Fini M, Giavaresi G, Setti S, et al. Cartilage repair with osteochondral autografts in sheep: effect of biophysical stimulation with pulsed electromagnetic fields. J Orthop Res. 2008;26:631-42. doi:10.1002/ jor.20530.

24. Adravanti P, Nicoletti S, Setti S, Ampollini A, de Girolamo L. Effect of pulsed electromagnetic field therapy in patients undergoing total knee arthroplasty: a randomised controlled trial. Int Orthop. 2014;38:397-403. doi:10.1007/s00264-013-2216-7.

25. Moretti B, Notarnicola A, Moretti L, Setti S, De Terlizzi F, Pesce V, et al. I-ONE therapy in patients undergoing total knee arthroplasty: a prospective, randomized and controlled study. BMC Musculoskelet Disord. 2012;13:88 doi:10.1186/1471-2474-13-88.

26. Nelson FR, Zvirbulis R, Pilla AA. Non-invasive electromagnetic field therapy produces rapid and substantial pain reduction in early knee osteoarthritis: a randomized double-blind pilot study. Rheumatol Int. 2013;33:2169-73. doi:10.1007/s00296-012-2366-8.

27. Thamsborg G, Florescu A, Oturai P, Fallentin E, Tritsaris K, Dissing S. Treatment of knee osteoarthritis with pulsed electromagnetic fields: a randomized, double-blind, placebo-controlled study. Osteoarthritis Cartilage. 2005;13:575-81.

28. Ay S, Evcik D. The effects of pulsed electromagnetic fields in the treatment of knee osteoarthritis: a randomized, placebo-controlled trial. Rheumatol Int. 2009;29:663-6. doi:10.1007/s00296-008-0754-x.

29. Ozgüçlü E, Cetin A, Cetin M, Calp E. Additional effect of pulsed electromagnetic field therapy on knee osteoarthritis treatment: a randomized, placebo-controlled study. Clin Rheumatol. 2010;29:927-31. doi:10.1007/s10067-010-1453-z.

30. Clutterbuck AL, Mobasheri A, Shakibaei M, Allaway D, Harris P. Interleukin-1 $\beta-$ Induced Extracellular Matrix Degradation and Glycosaminoglycan Release is Inhibited by Curcumin in an Explant Model of Cartilage Inflammation. Ann N Y Acad Sci. 2009;1171:428-35. doi:10.1111/j.1749-6632.2009.04687.x.

31. Stabellini G, De Mattei M, Calastrini C, Gagliano N, Moscheni C, Pasello M, et al. Effects of interleukin-1 beta on chondroblast viability and extracellular matrix changes in bovine articular cartilage explants. Biomed Pharmacother. 2003;57:314-9.

32. De Mattei M, Varani K, Masieri FF, Pellati A, Ongaro A, Fini M, et al. Adenosine analogs and electromagnetic fields inhibit prostaglandin E2 release in bovine synovial fibroblasts. Osteoarthritis Cartilage. 2009;17:252-62. doi:10.1016/ j.joca.2008.06.002.

33. Ongaro A, Pellati A, Setti S, Masieri FF, Aquila G, Fini M, et al. Electromagnetic fields counteract IL-1 $\beta$ activity during chondrogenesis of bovine mesenchymal stem cells. J Tissue Eng Regen Med. 2012. doi:10.1002/term.1671.

34. O'Driscoll SW, Keley FW, Salter RB. Durabilty of regenerated articular cartilage produced by free autogenous periosteal grafts in major full-thickness defects in joint surfaces under the influence of continuous passive motion. A follow-up report at one year. J Bone Joint Surg Am. 1988;70:595-606.

35. Vincent HK, Percival SS, Conrad BP, Seay AN, Montero C, Vincent KR. Hyaluronic Acid (HA) Viscosupplementation on Synovial Fluid Inflammation in Knee Osteoarthritis: A Pilot Study. Open Orthop J. 2013;7:378-84. doi:10.2174/1874325001307010378. eCollection 2013.

36. Blaney Davidson EN, van Caam AP, Vitters EL, Bennink MB, Thijssen E, van den Berg WB, et al. TGF- $\beta$ is a potent inducer of Nerve Growth Factor in articular cartilage via the ALK5-Smad2/3 pathway. Potential role in OA related pain? Osteoarthritis Cartilage. 2015;23:478-86. doi:10.1016/.joca.2014.12.005.

37. Li P, Raitcheva D, Hawes M, Moran N, Yu X, Wang F, et al. 20 maintains cartilage integrity and decreases osteophyte formation in osteoarthritis through both anabolic and anti-catabolic mechanisms. Osteoarthritis Cartilage. 2012;20:1336-46. doi:10.1016/j.joca.2012.07.004.

38. Stradner MH, Angerer H, Ortner T, Fuerst FC, Setznagl D, Kremser ML, et al. The immunosuppressant FTY720 (fingolimod) enhances glycosaminoglycan depletion in articular cartilage. BMC Musculoskelet Disord. 2011;12:279. doi:10.1186/1471-2474-12-279.

39. Kim J, Xu M, Xo R, Mates A, Wilson GL, Pearsall 4th AW, et al. Mitochondrial DNA damage is involved in apoptosis caused by pro-inflammatory cytokines in human OAchondrocytes. Osteoarthritis Cartilage. 2010;18:424-32. doi:10.1016/j.joca.2009.09.008.

40. Rai MF, Graeve T, Twardziok S, Schmidt MF. Evidence for regulated interleukin-4 expression in chondrocyte-scaffolds under in vitro inflammatory conditions. PLoS One. 2011;6:e25749. doi:10.1371/ journal.pone.0025749.

41. Clérigues V, Guillén MI, Gomar F, Alcaraz MJ. Haem oxygenase-1 counteracts the effects of interleukin-1 $\beta$ on inflammatory and senescence markers in cartilage-subchondral bone explants from osteoarthritic patients. Clin Sci (Lond). 2012;122:239-50. doi:10.1042/CS20100519.

42. Li P, Raitcheva D, Hawes M, Moran N, Yu X, Wang F, et al. Hylan G-F 20 maintains cartilage integrity and decreases osteophyte formation in osteoarthritis through both anabolic and anti-catabolic mechanisms. Osteoarthritis Cartilage. 2012;20:1336-46. doi:10.1016/j.joca.2012.07.004

43. Julovi SM, Yasuda T, Shimizu M, Hiramitsu T, Nakamura T. Inhibition of interleukin-1 beta-stimulated production of matrix metalloproteinases by hyaluronan via CD44 in human articular cartilage. Arthritis Rheum. 2004;50:516-25.

44. Brighton CT, Wang W, Clark CC. Up-regulation of matrix in bovine articular cartilage explants by electric fields. Biochem Biophys Res Commun. 2006;342:556-61.

45. Brighton $C T$, Wang W, Clark CC. The effect of electrical fields on gene and protein expression in human osteoarthritic cartilage explants. J Bone Joint Surg Am. 2008;90:833-48. doi:10.2106/JBJS.F.01437.

46. Lim EH, Sardinha JP, Myers S, Stevens M. Latent transforming growth factorbeta1 functionalised electrospun scaffolds promote human cartilage differentiation: towards an engineered cartilage construct. Arch Plast Surg. 2013:40:676-86. doi:10.5999/aps.2013.40.6.676.

47. Negm A, Lorbergs A, Macintyre NJ. Efficacy of low frequency pulsed subsensory threshold electrical stimulation vs placebo on pain and physical function in people with knee osteoarthritis: systematic review with meta-analysis. Osteoarthritis Cartilage. 2013;21:1281-9. doi:10.1016/ j.joca.2013.06.015.

48. Clutterbuck AL, Smith JR, Allaway D, Harris P, Liddell S, Mobasheri A. High throughput proteomic analysis of the secretome in an explant model of articular cartilage inflammation. J Proteomics. 2011;74:704-15. doi:10.1016/ j.jprot.2011.02.017.

49. Sztrolovics R, Recklies AD, Roughley PJ, Mort JS. Hyaluronate degradation as an alternative mechanism for proteoglycan release from cartilage during interleukin-1 beta-stimulated catabolism. Biochem J. 2002;362(Pt 2):473-9.

50. Hargrave-Thomas EJ, Thambyah A, McGlashan SR, Broom ND. The bovine patella as a model of early osteoarthritis. J Anat. 2013;223:651-64. doi:10.1111/joa.12115.

\section{Submit your next manuscript to BioMed Central and take full advantage of:}

- Convenient online submission

- Thorough peer review

- No space constraints or color figure charges

- Immediate publication on acceptance

- Inclusion in PubMed, CAS, Scopus and Google Scholar

- Research which is freely available for redistribution 